\title{
CULTIVO DE PAICHE, Arapaima gigas, UTILIZANDO BUJURQUI, Cichlassoma bimaculatum, COMO PRESA
}

Fernando A lcántara Bocanegra ( $\left.{ }^{*}\right)$

Humberto Guerra Flores $\left({ }^{*}\right)$

\section{RESUMEN}

En éste trabajo se reportan los resultados del cultivo de paiche, Arapaima gigas, utilizando bujurqui, Cichlassoma bimaculatum, como presa, en un estanque semi natural de 4,900 $\mathrm{m}^{2}$.

La siembra del bujurqui se efectuó ciento veinte días antes que la siembra del paiche, con una carga de 10,000 peces/Ha. y con una longitud promedio de $7.0 \mathrm{~cm} \mathrm{La}$ siembra del paiche se efectuó a una carga de 44 peces $/ \mathrm{Ha}$., con una longitud de $45 \mathrm{~cm}$. y845g.

A los catorce meses de cultivo, los paiches alcanzaron una longitud de $73.7 \mathrm{~cm}$. y 3,468 g., con una biomasa de $72.8 \mathrm{~kg} /$ estanque, lo que significa $145 \mathrm{~kg} / \mathrm{ha}$.

Palabras claves: Cultivo Predador-Presa, Arapaima gigas Cichlassoma bimaculatus

\section{INTRODUCCION}

El paiche, Arapaima gigas (Cuvier, 1829), es un pez de gran demanda en la amazonia peruana, cuyas posibilidades de cultivo han sido poco exploradas, fundamentalmente, por su régimen carnívoro.

El conocimiento de la bioecología de la especie es más o menos amplio y está basado mayormente en observaciones en el medio natural. Entre otros, Fontenele (1959), en Brasil, reportó los hábitos de desove y evolución de la larva, así como efectuó estudios de la bioecología de la especie. En el Perú, Sánchez (1960), estudió los aspectos de su historia natural, ecología y aprovechamiento; Berger (1972), estudió el factor de condición; Copaira y M ontalvo (1972), trataron aspectos de dimorfismo sexual en base a estudios de coloración; Tresierra (1976), estudió la biología y ecología; C Guerra (1980), estudió el desarrollo sexual y ciclo de maduración.

A unque el paiche, es un pez de régimen carnívoro, en cautiverio no exige presas vivas y acepta trozos de carne de pescado. Su gran tamaño hace pensar que es un

INSTITUTO DE INVESTIGACIONES DE LA AMAZONIA. Av. A belardo Quiñónez Km.2.5.Apartado 784. Iquitos - Perú 
pez, más de piscicultura extensiva que intensiva, pudiendósele emplear en grandes estanques con un pez forraje (Bard, et al. 1975).

Este trabajo tuvo como objetivo evaluar la producción de paiche, Arapaima gigas, en cultivo predador-presa, con el ciclido nativo, Cichlassoma bimaculatum.

\section{MATERIAL Y METODOS}

Este cultivo se realizó en un estanque de 4,900 $\mathrm{m}^{2}$ de espejo de agua y profundidades comprendidas entre $0.20 \mathrm{~m}$. y $1.80 \mathrm{~m}$.

Los paiches procedieron de la primera reproducción en cautiverio de una pareja, luego de seis años de cría (Alcántara, 1991), y los bujurquis procedieron de reproducciones natural es ocurridas en un estanque donde aparecieron como invasores.

La siembra del bujurqui se efectuó cuatro meses antes de la introducción del paiche, con el fin de que tuvieran el tiempo necesario para que se establezca plenamente en el estanque. En este momento, el paiche tuvo $45 \mathrm{~cm}$. y $845 \mathrm{gr}$. de longitud y peso promedio respectivamente y seis meses de edad. El bujurqui, en cambio tuvo $7 \mathrm{~cm}$. y 7 g., de longitud y peso promedio, respectivamente.

$\mathrm{L}$ a tasa de siembra del bujurqui fue de $1 \mathrm{pez} / \mathrm{m}^{2}$ y del paiche de $1 \mathrm{pez} / 222 \mathrm{~m}^{2}$

El estanque fue fertilizado con gallinaza en una tasa de $37 \mathrm{~kg} / \mathrm{ha} /$ aplicación. $\mathrm{L}$ a administración se efectuó tres veces por semana espolvoreando el fertilizante desde las orillas.

Los muestreos de crecimiento del paiche se efectuaron mensualmente, abarcando en cada ocasión más del $20 \%$ de la población sembrada.

Durante la ejecución del experimento se registraron los parámetros limnológicos siguientes: oxígeno disuelto, anhidrido carbónico, temperatura y $\mathrm{pH}$.

Se calculó la curva de crecimiento en longitud para el paiche, de acuerdo al modelo de von Bertalanffy (1938), adaptado por Santos (1978) para cultivo intensivo:

\section{$L i=L 8 \quad\left\{1-e^{-k(T+T e)}\right\}$}

Donde:

LT = L ongitud total media de los individuos.

L8 = L ongitud total máxima que pueden alcanzar los individuos, en promedio, en condiciones de cultivo.

$\mathrm{E}=$ Base de los logaritmos neperianos.

$\mathrm{K}=$ Parámetro relacionado con la velocidad de crecimiento de la especie.

$1=$ Tiempo de cultivo.

Te $\quad=$ Factor de corrección del tiempo de cultivo $(\mathrm{T})$, relacionado con la longitud total media de los peces en el instante de la siembra (Le). 


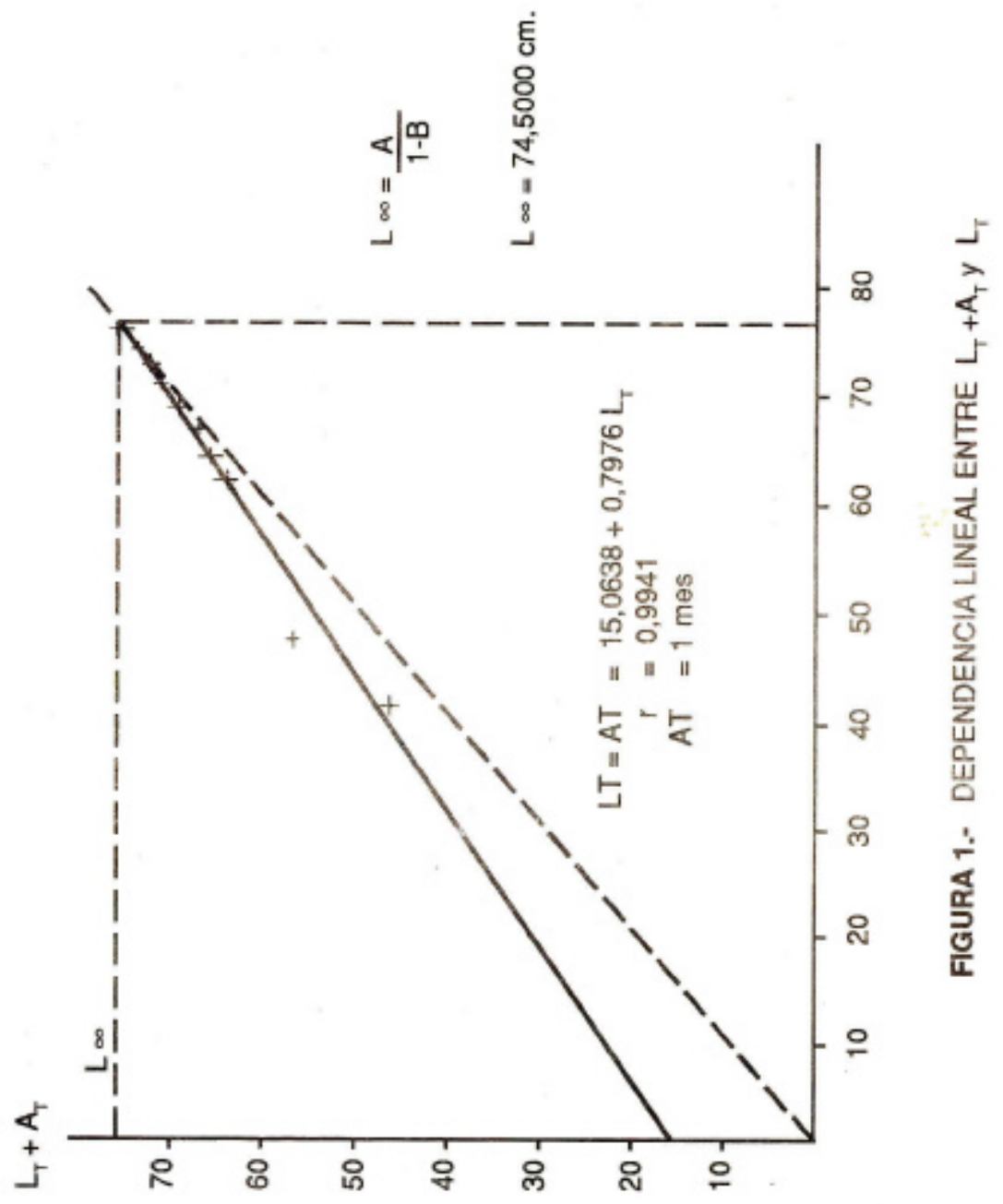



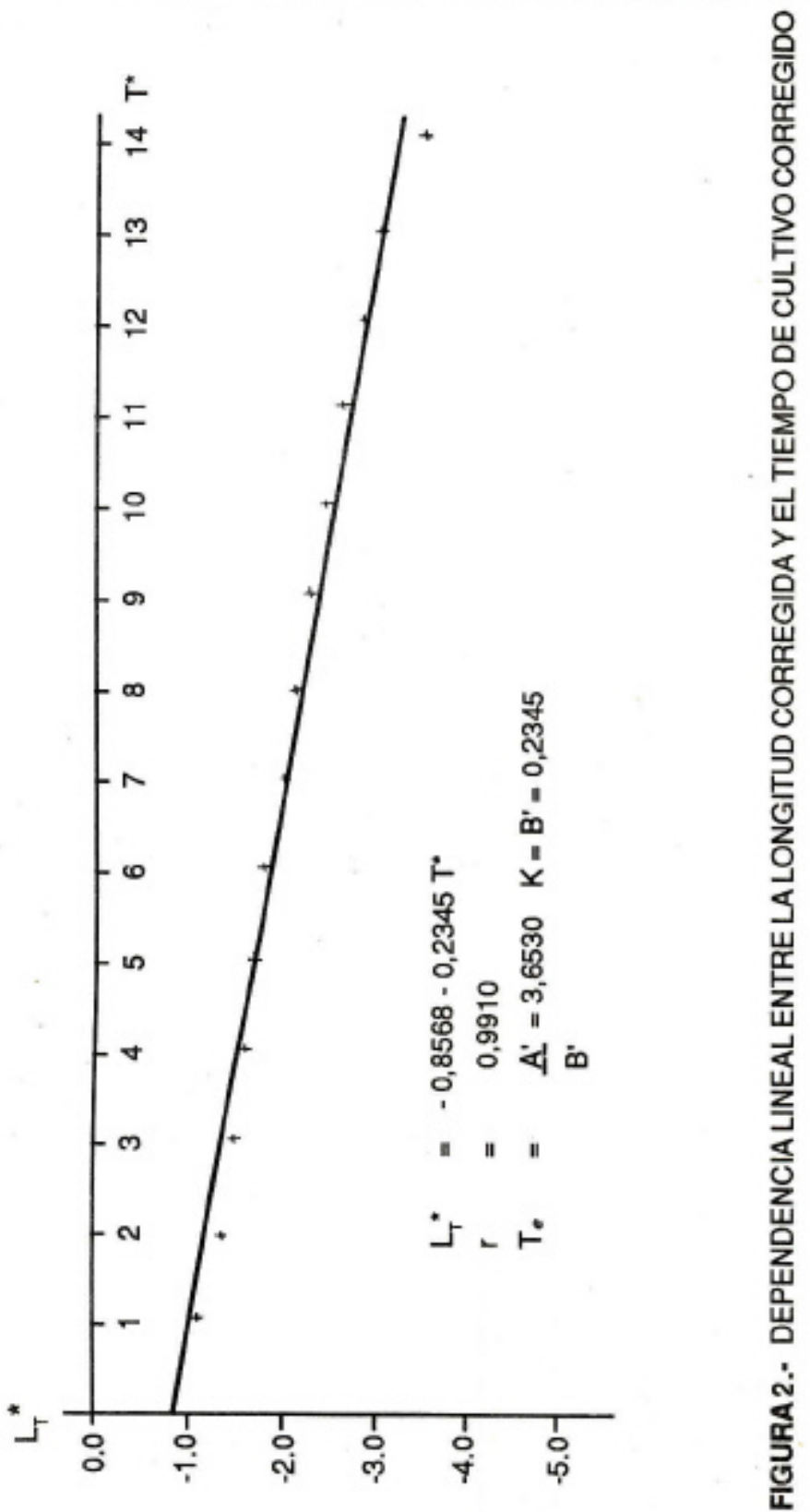


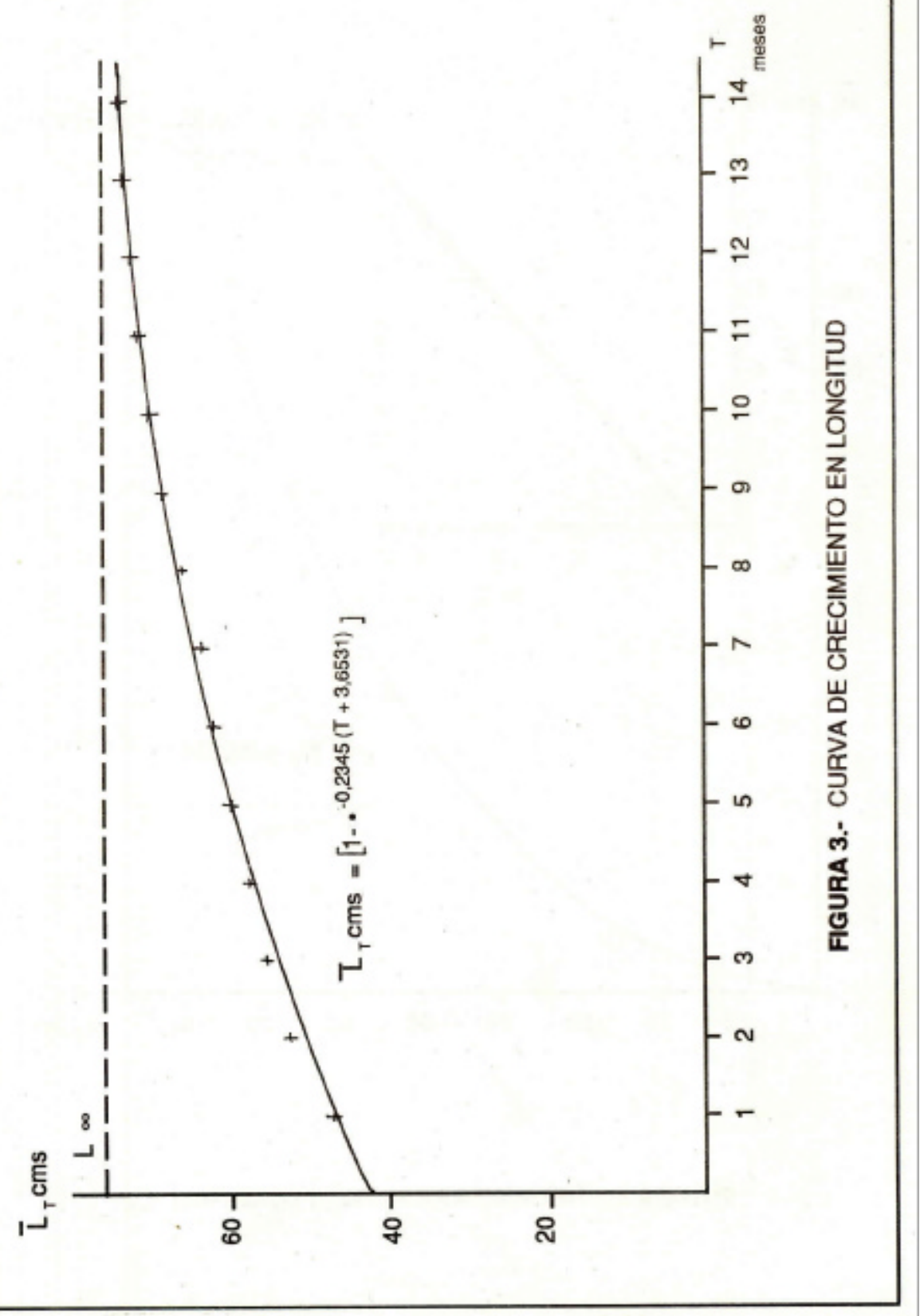




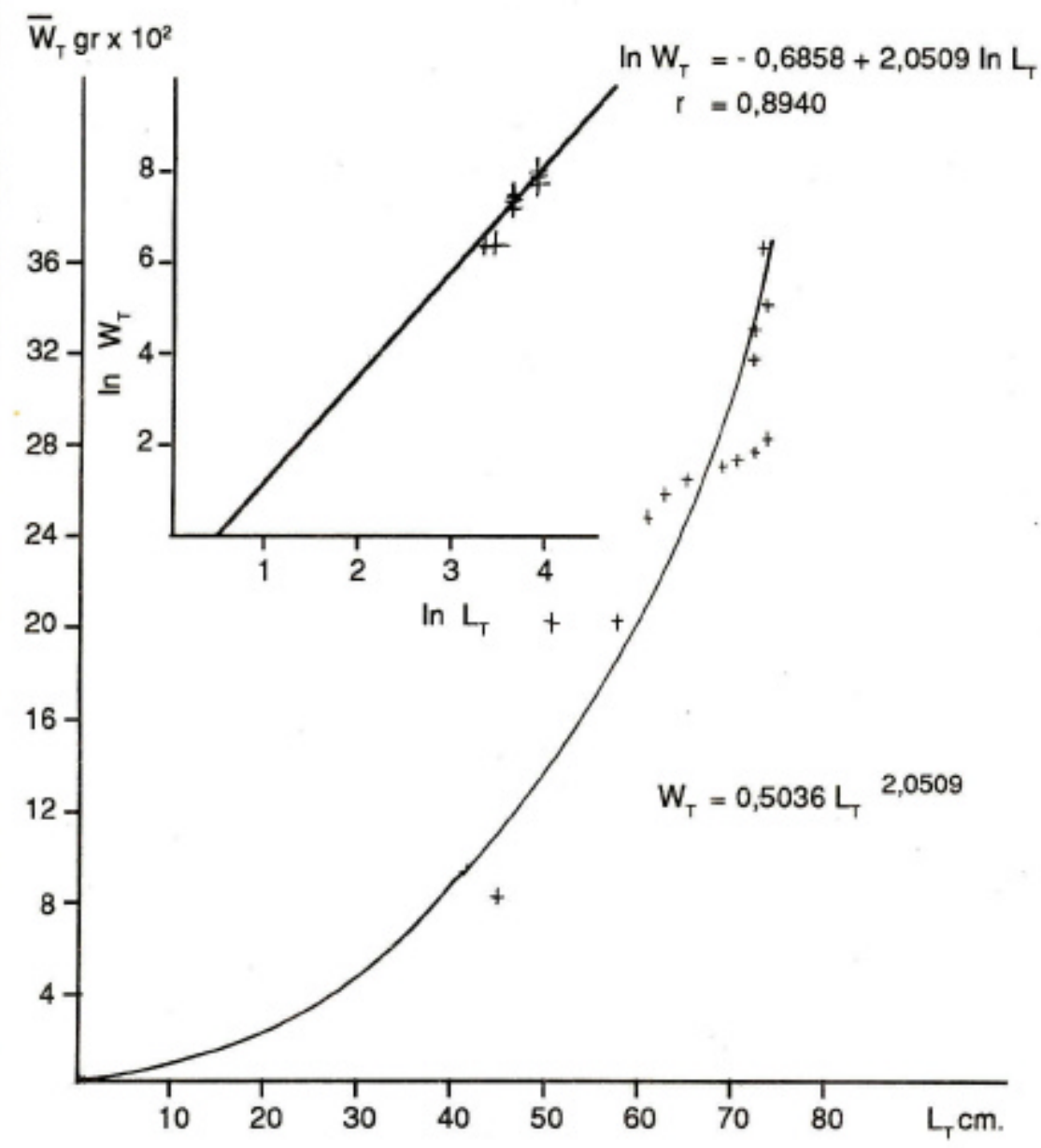

FIGURA 4.- RELACION PESO $\left(\mathrm{W}_{\mathrm{T}}\right)$ / LONGITUD (L $)$ 


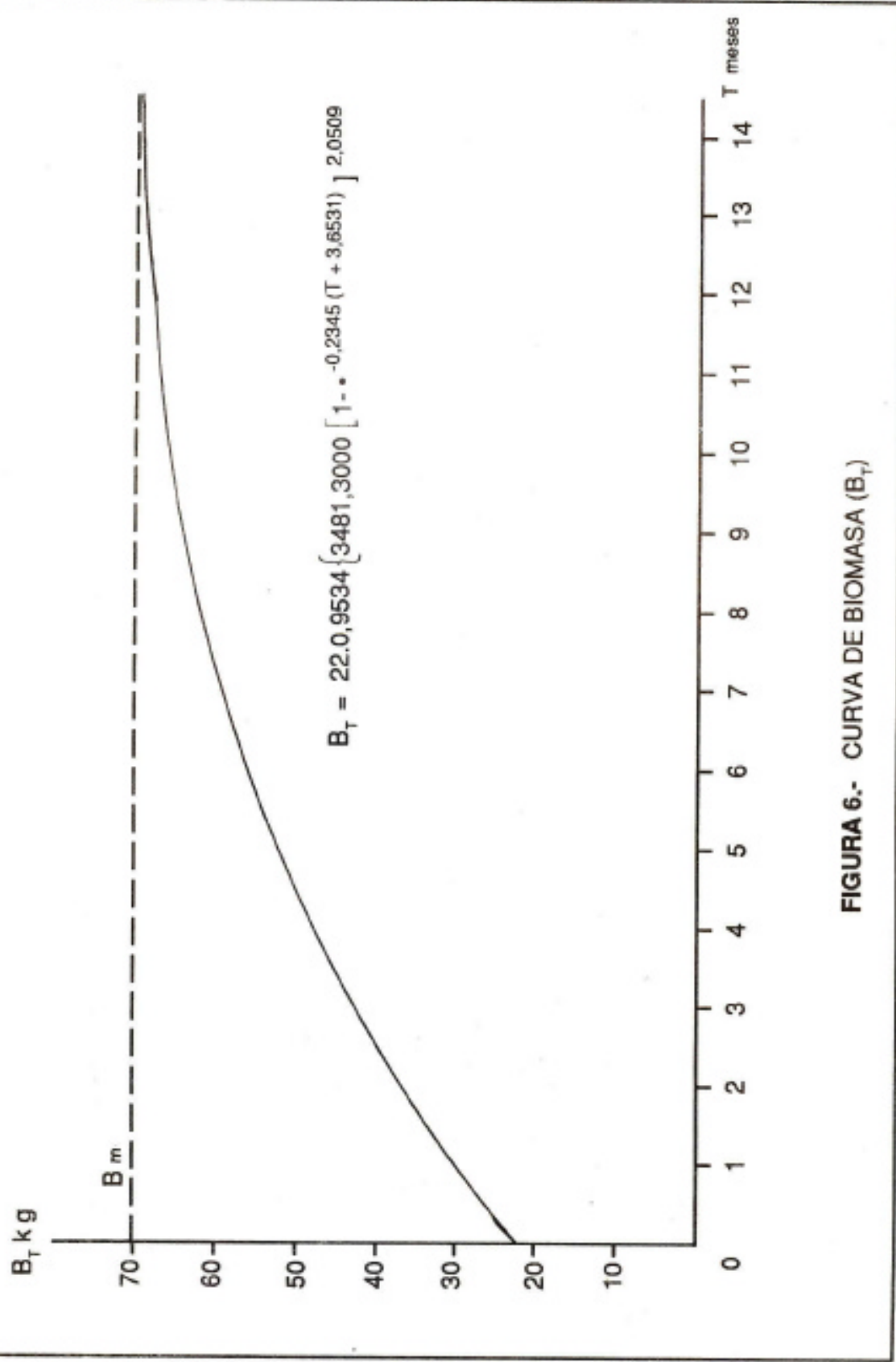




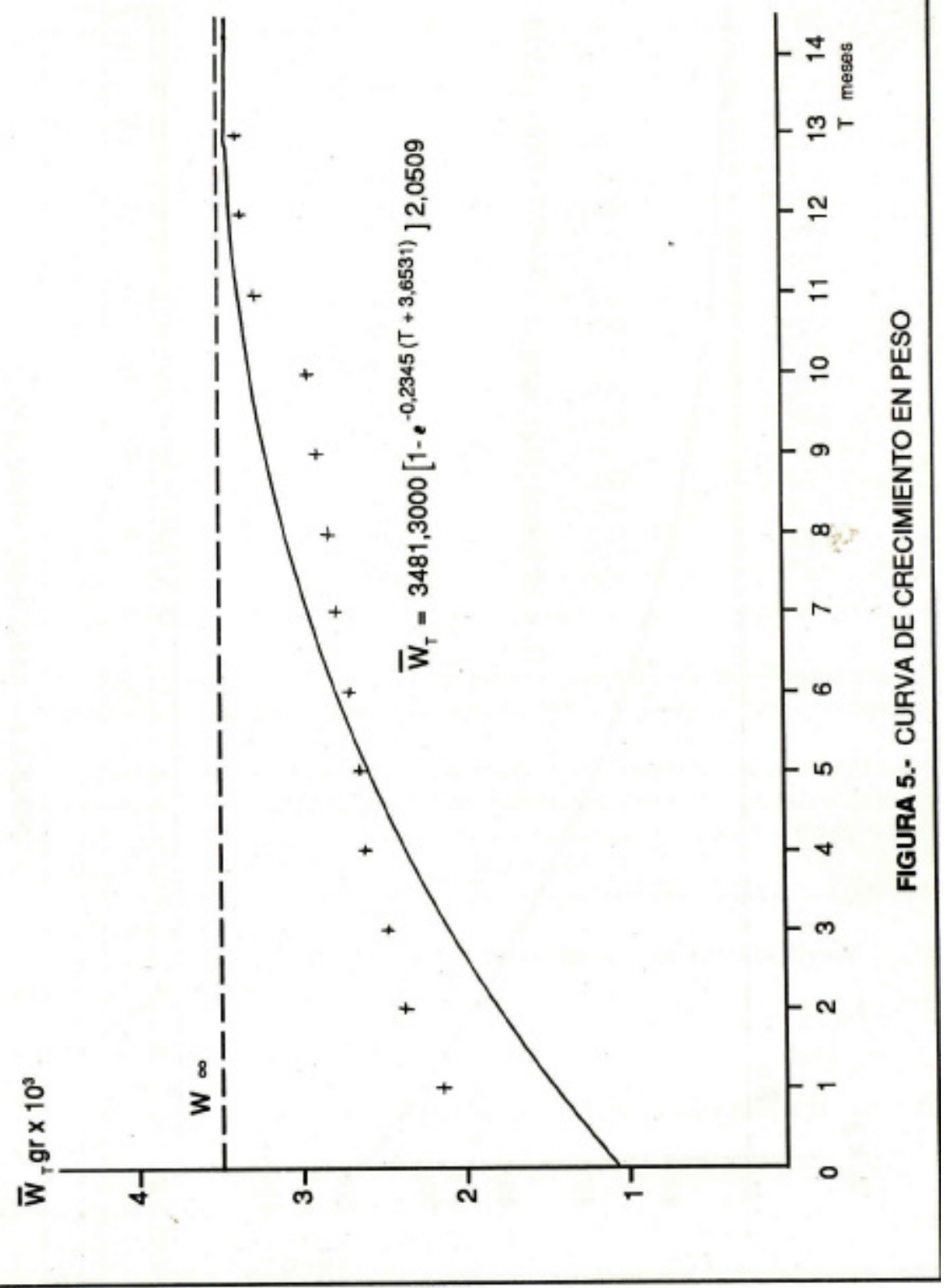




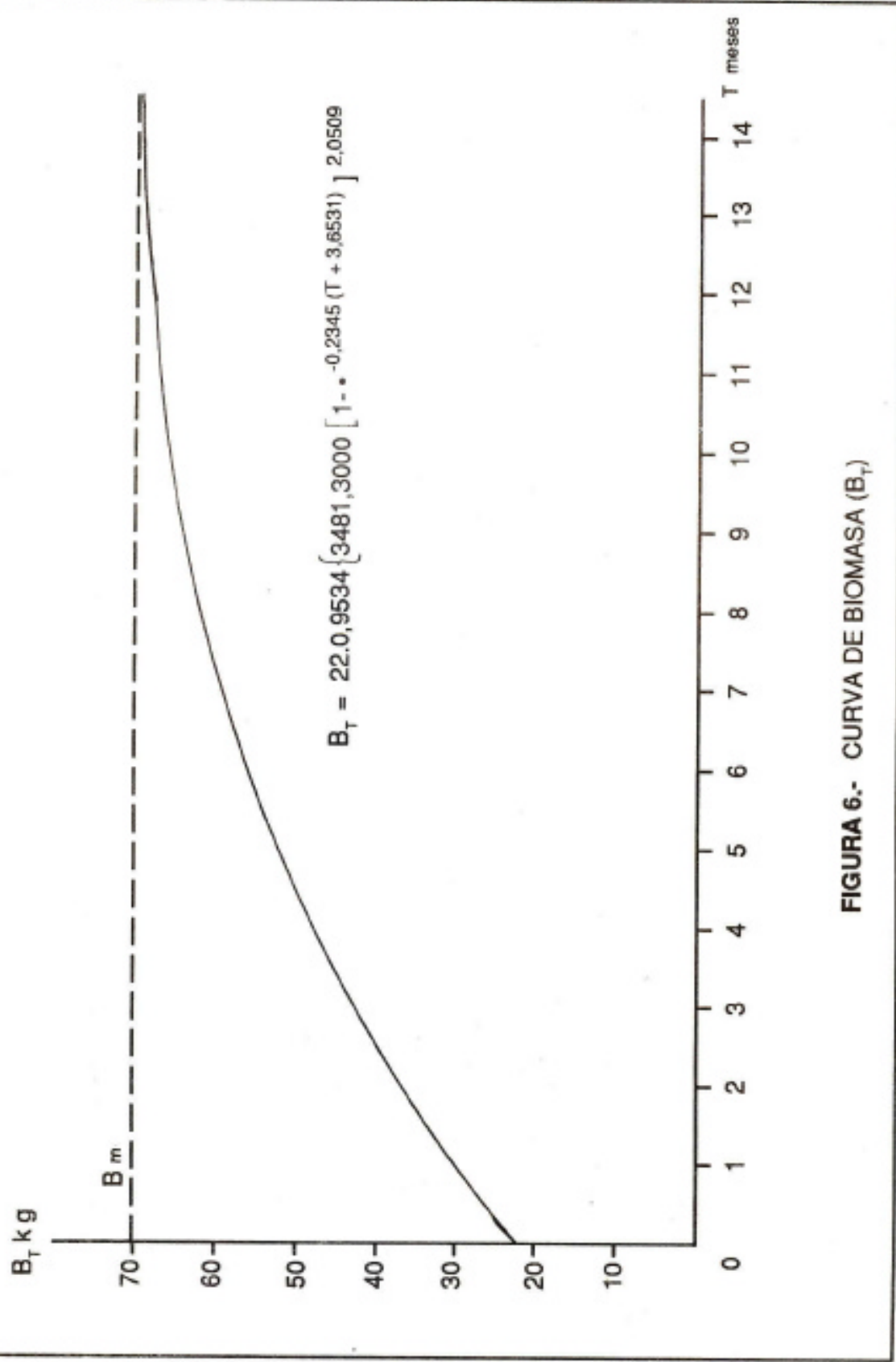




\section{RESULTADOS}

TABLA 1 : LONGITUD Y PESO PROMEDIO OBSERVADOS A TRAVES DEL PERIODO DE CULTIVO

\begin{tabular}{|cccc|}
\hline $\begin{array}{c}\text { T } \\
\text { Meses }\end{array}$ & $\begin{array}{l}\text { LT } \\
\mathrm{cm}\end{array}$ & $\begin{array}{c}\text { WT } \\
\mathrm{g}\end{array}$ & $\begin{array}{l}\text { BT } \\
\mathrm{Kg}\end{array}$ \\
\hline 0 & 45,0 & 845 & 18.5 \\
1 & 50,0 & 2,104 & 46.2 \\
2 & 57,2 & 2,360 & 51.9 \\
3 & 60,0 & 2,506 & 55.1 \\
4 & 62,4 & 2,635 & 55.3 \\
5 & 64,4 & 2,687 & 56.4 \\
6 & 66,3 & 2,750 & 57.7 \\
7 & 67,8 & 2,790 & 58.5 \\
8 & 69,0 & 2,790 & 58.5 \\
9 & 70,0 & 2,803 & 58.8 \\
10 & 71,0 & 2,864 & 60.1 \\
11 & 71.8 & 3,160 & 66.3 \\
12 & 72,5 & 3,207 & 67.3 \\
13 & 73.1 & 3,275 & 68.7 \\
14 & 73,7 & 3,468 & 72.8 \\
\hline
\end{tabular}

La Tabla 1 muestra que los paiches que se sembraron con una longitud de 45.0 $\mathrm{cm}$. y $845 \mathrm{~g}$., alcanzaron un promedio $73.7 \mathrm{~cm}$. y 3,468 g. de longitud y peso respectivamente.

La Figura 1, muestra la dependencia lineal entre los parámetros $L_{T}+T y L_{T}$, con la cual se comprobó la validéz del modelo de von Bertalanffy para obtener la curva de crecimiento en longitud de la especie en estudio. La longitud máxima calculada ( 8 ) para el paiche en las condiciones de cultivo fue de $74.5 \mathrm{~cm}$.

A simismo en la Fig. 2 se muestra la dependencia lineal entre la longitud corregida y el tiempo de cultivo corregido.

La curva de crecimiento en longitud obtenida se muestra en la Figura 3 y su expresión matemática es:

$$
L_{T}=74,5000\{1-e-0,2345(T+3,6531)\}
$$

Al plotearse los puntos empfricos, se verificó alta adherencia a la curva de ajustamiento.

La Figura 4 muestra la relación entre el peso (Wí) y la longitud $\left(L_{T}\right)$. La expresión matemática de la recta y la curva calculadas es:

$\ln W_{T}=-0,6858+2,0509 \ln L_{T}$ 
$W_{T}=0,5036 L_{T} 2,0509$

Del mismo modo, los puntos empíricos muestran alta adherencia a la recta ya la curva de ajustamiento, respectivamente. Reemplazando la longitud total $\mathrm{Li}$ por la longitud máxima en la relación peso-longitud, se calculó el peso máximo $\mathrm{W} 8=3481,3 \mathrm{gr}$.

En la Figura 5, se muestra la curva de crecimiento en peso del paiche y su expresión matemática es:

$W_{T}=3481,3000(1-8-0,2345(1+3,6531)) 2,0509$

Al plotear los puntos empíricos se verificaron buena adherencia a la curva de ajustamiento.

En la Figura 6 se muestra la curva de biomasa ( $\mathrm{Br}$ ), cuya expresión matemática es:

$\mathrm{B}_{\mathrm{T}}=22.0,9534(3481,300011-8-0,2345(1+3,6531) 2,0509\}$

L a tasa de sobrevivencia total calculada (S*) fue de $95 \%$, con una tasa mensual $\left(S^{*}{ }_{T}\right.$ ) de 0,9966 . El coeficiente de mortalidad (M) calculado fue de 0,0034 .

La biomasa máxima calculada fue de $71.1 \mathrm{~kg} / \mathrm{estanque}$, 10 que significa $145 \mathrm{~kg} / \mathrm{ha}$; asimismo, el tiempo de biomasa máxima calculado fue de 24.8 meses, con un índice de rendimiento de 2.86. Finalmente, los factores físicos y químicos variaron entre límites normales, como se muestra en la Tabla 2.

TABLA 2.- VARIABLES LIMNOLOGICAS DEL ESTANQUE

\begin{tabular}{|c|c|c|c|c|c|c|}
\hline $\begin{array}{l}\text { To C } \\
\text { A tm }\end{array}$ & $\begin{array}{l}\text { T으 } \\
\text { A gua }\end{array}$ & $\begin{array}{l}\text { D.S } \\
\mathrm{cm}\end{array}$ & $\mathrm{pH}$ & $\begin{array}{l}\text { Conduct. } \\
\text { Elect. K } 25 \\
\text { umhos/cm }\end{array}$ & $\begin{array}{l}\text { Oxig. } \\
\text { Dis. } \\
\text { Ppm }\end{array}$ & $\begin{array}{l}\mathrm{CO}_{2} \\
\mathrm{Libre} \\
\mathrm{ppm}\end{array}$ \\
\hline $\begin{array}{l}R 23-32.8 \\
\times 28,4\end{array}$ & $\begin{array}{l}25,7-34,8 \\
29,6\end{array}$ & $\begin{array}{l}32-105 \\
52,4\end{array}$ & $\begin{array}{l}5,0-9,5 \\
6,5\end{array}$ & $\begin{array}{l}10,2-33,0 \\
19,3\end{array}$ & $\begin{array}{l}4,5-10,6 \\
8,0\end{array}$ & $\begin{array}{l}0,0-4,0 \\
0,9\end{array}$ \\
\hline
\end{tabular}

\section{DISCUSION}

La longitud máxima de $74,5 \mathrm{~cm}$. y el peso máximo de $3481,3 \mathrm{~g}$. calculados para la especie en estudio se consideran bajos y se atribuye al posible desbalance entre prcdador y presa originado por el ataque del paiche al bujurqui en las zonas de agua superficial de las orillas del estanque, lugares que eran utilizados por los bujurquis para la ubicación de sus nidos y cría de las larvas y alevinos. Por consiguiente, la biomasa máxima de $145 \mathrm{~kg} / \mathrm{ha}$ calculada, es también baja, aún cuando se trata de una especie de régimen alimenticio carnívoro. 
El tiempo de biomasa máxima calculado de 24,8 meses, sugiere que la suspensión del cultivo a los 14 meses fue oportuno, ya que a este período el incremento de biomasa fue mínimo y tendria hacia la asíntota.

Finalmente, el índice de rendimiento de 2,86 calculado, es sumamente bajo. Sin embargo, se considera que este índice podría elevarse protegiendo las zonas de reproducción de la población presa, así como intensificando la tasa de fertilización 0 asociando este tipo de cultivo a la cría de cerdos, para favorecer la producción de alimento para la población presa. O tra alternativa sería bajar la tasa de carga del paiche.

\section{BIBLIOGRAFIA}

ALCANTARA, B.F. 1991. Observaciones sobrecomportamiento reproductivo de paiche, Arapaima gigas, en cautiverio. Folia A mazón Vol.2. (165-168) Instituto de Investigaciones de la A mazonía Peruana. Iquitos - Perú.

BARD, J.; P. DE KIMPE; J.LEMASON; P.LESSENT. 1975. M anual de piscicultura destinado a la A mérica Tropical. 2da. Ed. Centre Technique Forestier Tropical. Francia.

COPAIRA, M. y C. MONTALV 0. 1972. Dimorfismo sexual en el paiche (Arapaima gigas, Cuvier, 1829). Rey. Inv.Pes. (IVITA), (2) 203-207. Univ. Nac. 5. M arcos. Lima - Perú.

BERGER, C. 1972. Estudio del factor de condición del paiche Arapaima gigas (Cuvier) en la Zona Reservada del río Pacaya entre los años 1959 a 1969. Tesis Bach. Cienc. UN M SM. Lima. Perú.

FONTENELE, 0. 1959.a. Hábitos de desova do pirarucu Arapaima gigas (Cuvier) (Pisces:

Isospondyli, A rapaimidae), e evolucao de sua larva. Coletanea de Trabalhos Tecnicos. Publicacao № 153. IC.

959. b. Contribucao para o conhecimento debiología do pirarucu, Arapaima gigas (Cuvier) em cativeiro (A ctinopterigii, osteoglossidae). Coletanea de Trabalhos Tecnicos. IC -166: - 235-250.

GUERRA, H. 1980. Desarrollo sexual del paiche, Arapaima gigas, en las Zonas Reservadas del Estado (Ríos Pacaya y Samiria) 1971-1975. Inf. N 2 67. Inst. Mar del Perú. Callao. Perú.

SANCHEZ, J. 1960. El paiche. A spectos de su historia natural. Ecología natural. Ecología y aprovechamiento. Rey. Caza y Pesca № 10. Dirección de Pesquería y Caza. M inisterio de A gricultura. Lima-Perú.

SANTOS, E.P. dos. 1978. Dinámica de populacoes aplicada a pesca e a piscicultura. Sao Paulo, Ed. da U niversidade de Sao Paulo. 129 p.

TRESIERRA, A. 1976. Algunos aspectos de la Biología y Ecología del "paiche" Arapaima gigas $C$. del lago Sauce (Dpto. San Martín). Tesis Doctoral Ciencias Biológicas. UNT. 35pp. 